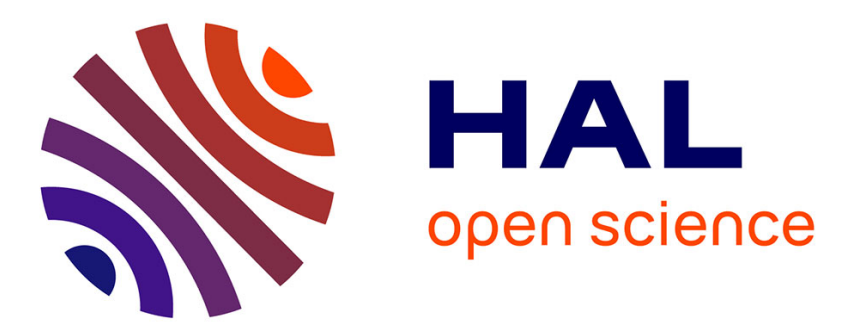

\title{
CORDE FROTTÉE: TYPE PARTICULIER DE MOUVEMENT A DEUX VITESSES OBSERVÉ SOUS L'ARCHET
}

R. Caussé, S. Radulovic, G. Weinreich

\section{- To cite this version:}

R. Caussé, S. Radulovic, G. Weinreich. CORDE FROTTÉE: TYPE PARTICULIER DE MOUVEMENT A DEUX VITESSES OBSERVÉ SOUS L'ARCHET. Journal de Physique IV Proceedings, 1992, 02 (C1), pp.C1-47-C1-50. 10.1051/jp4:1992106 • jpa-00251048

\section{HAL Id: jpa-00251048 https://hal.science/jpa-00251048}

Submitted on 1 Jan 1992

HAL is a multi-disciplinary open access archive for the deposit and dissemination of scientific research documents, whether they are published or not. The documents may come from teaching and research institutions in France or abroad, or from public or private research centers.
L'archive ouverte pluridisciplinaire HAL, est destinée au dépôt et à la diffusion de documents scientifiques de niveau recherche, publiés ou non, émanant des établissements d'enseignement et de recherche français ou étrangers, des laboratoires publics ou privés. 


\title{
CORDE FROTTEE : TYPE PARTICULIER DE MOUVEMENT A DEUX VITESSES OBSERVE SOUS L'ARCHET
}

\author{
R. CAUSSE, S. RADULOVIC* et G. WEINREICH** \\ Institut de Recherche et Coordination Acoustique Musique, 31 rue Saint-Merri, F-75004 Paris, \\ France \\ ${ }^{*}$ T.U. Eindhoven, Postbus 513, NL-5600 MB Eindhoven, The Netherlands \\ ${ }^{* *}$ Randall Laboratory, University of Michigan, Ann Arbor, Michigan 48109, USA
}

Résumé - Des mouvements de la corde sous l'archet pour lesquels le point représentatif alterne plus d'une fois au cours d'un cycle entre deux vitesses ont été observés. Les conditions à satisfaire pour les obtenir sont étudiées à partir d'une simulation informatique de la corde frottée.

\begin{abstract}
Motions of the bowed string at the point where the bow is located were observed. The satisfying conditions to obtain these motions are studied with a computer simulation of the bowed string.
\end{abstract}

\section{1 - INTRODUCTION}

L'archet peut entretenir des mouvements de la corde (régimes) tels que le point de la corde représentatif de l'archet alterne entre deux vitesses constantes plus d'une fois au cours d'un cycle. Le mouvement à deux vitesses, le plus simple et le plus important en musique, est le mouvement de Helmholtz [1] pour lequel l'alternance entre deux vitesses est déterminé par la position de l'archet sur la corde; si $\beta \mathrm{L}$ et $(1-\beta) \mathrm{L}$ sont les deux longueurs de division de la corde, le point représentatif de l'archet aura une vitesse constante pendant la fraction $\beta$ du cycle (phase glissée ) et l'autre vitesse constante pendant la fraction (1- $\beta$ ) du cycle (phase adhérence ).

Ces régimes d'oscillation soulèvent des problèmes importants et fascinants pour le physicien; ils font parti des mouvements possibles inventoriés par Raman [2] dans son étude importante qui avait pour but, à l'origine, de donner la description cinématique la plus simple des mouvements particuliers de la corde frottée mesurés par Krigar-Menzel et Raps [3] à la suite des travaux de Helmholtz. Cependant, aucune observation n'avait été réalisée jusqu'ici au point de frottement, vu la difficulté de mesurer sous 1'archet. L'archet équivalent, développé par Caussé et Weinreich [5], a permis de lever cette difficulté.

Il est important de remarquer que le type de mouvement "double-phase de glissement" [4], bien connu des instrumentistes, est inclus dans ces types. Tous ces régimes qui, musicalement, sont généralement indésirables, peuvent être obtenus bien entenđu par les débutants mais aussi par les instrumentistes avertis lorsqu'ils jouent aux limites du jeu "normal".

\section{2 - CONDITIONS D'OBTENTION}

Deux de ces régimes sont visualisées à la figure 1 (deux périodes du déplacement et de la vitesse correspondante au point de frottement; $\beta=\mathrm{L} / 5$ ). Ici l'alternance des vitesses se fait trois fois au cours d'un cycle.

figure 1
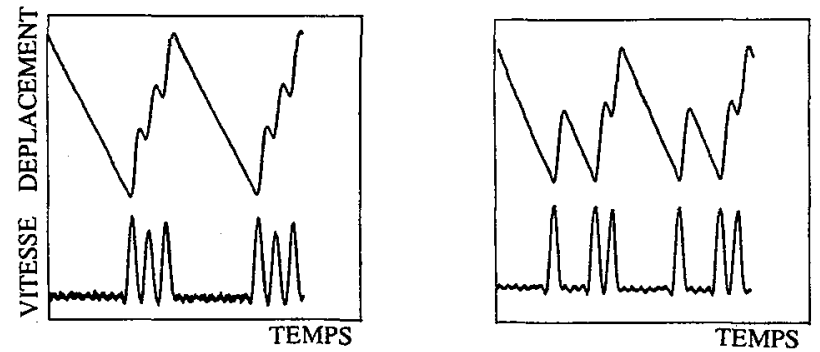
Ces types de mouvement nécessitent pour être obtenus et entretenus, des "pressions" d'archet minimales supérieures à celles des mouvements simples à deux vitesses; pour déloger la corde de ce régime stable de vibration et retrouver un régime plus simple, il faut soit diminuer la "pression" d'entretien, soit initialiser une nouvelle phase transitoire sur la corde.

Notons que ces mouvements sont obtenus plus ou moins facilement pour toute position d'archet (division aliquote ou pas de la corde).

De plus la durée totale des fractions du cycle passées à la première vitesse est conservée par rapport à un mouvement simple à deux vitesses. Il en est de même pour les fractions du cycle passées à l'autre vitesse.

Nous avons remarqué qu'un moyen pratique d'obtention de ces mouvements, lorsqu'un mouvement simple est établi sur la corde, consiste à perturber ce dernier dans le régime non-línéaire au moyen d'un générateur d'impulsions mécaniques. Mais l'apparition de ces mouvements ne se fait pas uniquement lorsque la perturbation est grande par rapport au mouvement, et parfois, une simple inversion du sens de l'archet est suffisante. Cependant, dans les deux cas, il est impossible de prévoir le régime obtenu car on ne contôle que la position où sera générée l'impulsion dans le premier cas et l'instant de commutation du sens de l'archet dans le deuxième, sans se préccuper de l'état de la corde à ce moment-là. Ceci nous a amené à réaliser une simulation entièrement informatique de la corde frottée.

\section{3 - TENTATIVE DE PRÉVISION ET SIMULATION INFORMATIQUE}

Pour les cas ou l'archet est appliqué en un point de division aliquote de la corde, il est possible de donner, par simple construction graphique, toutes les formes de vibrations possibles mais sans prévoir à l'avance celle qui sera obtenue. En effet, pour une division rationnelle de la corde par l'archet, la série des partiels qui ont un nœud en ce point est complètement absente dans la vibration résultante. Dans le cas de la figure 1, l'archet est situé au 1/5ème, ce qui veut dire à un noud de l'harmonique 5 et de ses multiples. Pour reproduire les deux formes représentées sur cette figure, on divise la phase glissée du mouvement simple correspondant à cette position d'archet en trois parties égales sur la courbe de la vitesse, et on répartit ces trois parties selon toutes les positions qui permettent de conserver la relation de phase initiale de chacune avec le 5 ème harmonique. On obtient toutes les formes pour lesquelles la phase glissée est divisée en trois (figure 2).

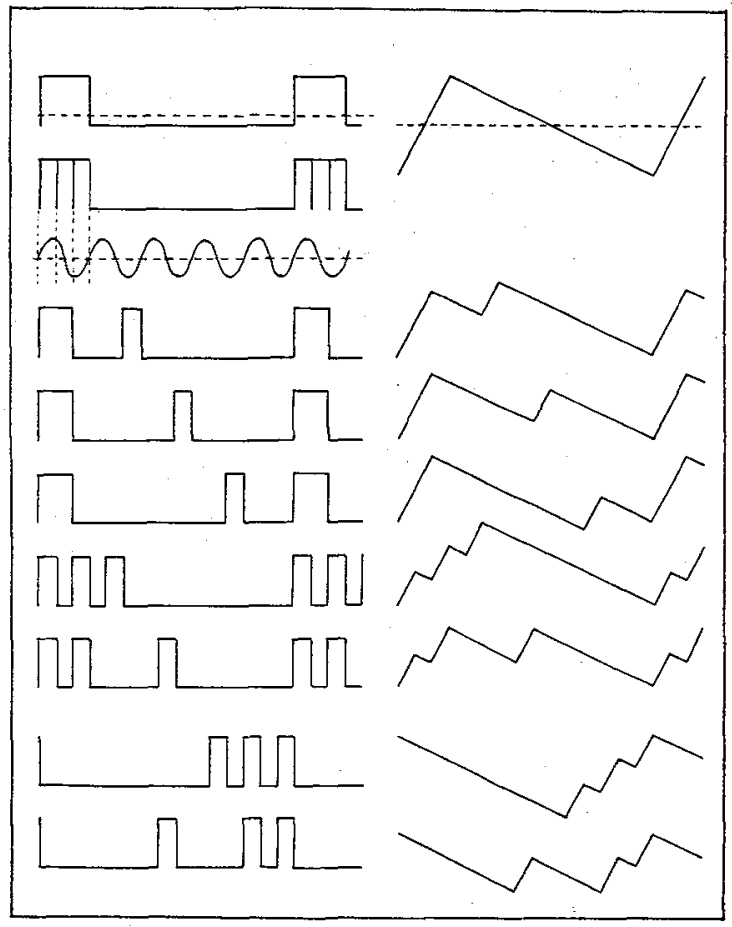

Cette opération doit être recommencée pour toutes les autres divisions de la phase glissée si l'on souhaite obtenir toutes les formes de division.

Pour mieux prévoir, parmi toutes les formes possibles, celle qui sera obtenue, nous avons utilisé une simulation informatique de notre archet équivalent. Le déclenchement du générateur d'impulsions est contrôlé par l'état de la corde à chaque instant. Dans le cas du mouvement d' Helmholtz, la décision se fait par rapport au coin qui se déplace sur la corde. Nous avons ainsi pu mettre en évidence la dépendance de l'état obtenu à l'état initial de la corde.

Un avantage supplémentaire de la simulation est de pouvoir suivre le trajet de l'impulsion et ses multiples réflexions jusqu'à l'obtention d'un mouvement stable.

figure 2 
La règle d'obtention suivante s'est dégagée des multiples simulations :

1. Pour obtenir ce type de mouvement, il est nécessaire d'avoir au point de frottement au moins 1 pic $>0$ pendant la phase glissée.

(convention: la vitesse, pendant la phase glissée est $<0$ et donc $>0$ pendant la phase adhérence).

2. Ce qui précède n'est pas suffisant; pour chaque pic $>0$ pendant la phase glissée, il faut un pic $<0$ pendant la phase adhérence.

Remarque: pic $>0$ pendant la phase adhérence et pic $<0$ pendant la phase glissée disparaissent toujours.

\section{4 - THÉORIE SIMPLIFIÉE}

Ecrivons :

a) les conditions de réflexion et de transmission des ondes au niveau de l'archet :

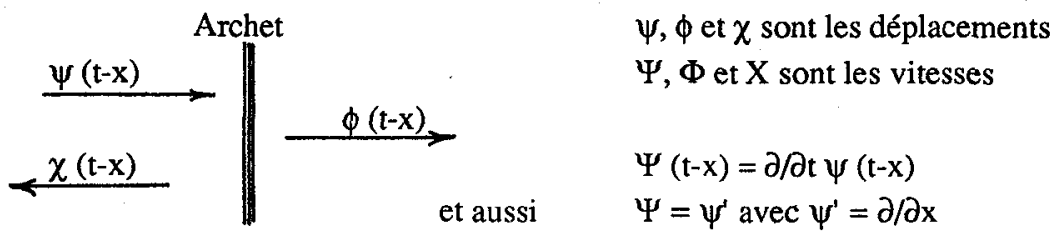

b) les conditions au niveau de l'archet liées :

- à la continuité:

$\psi(t-0)+\chi(t-0)=\phi(t-0)$

$$
\begin{array}{ll}
\text { d'ou } & \Psi+\chi=\phi \\
\text { et } & \Psi+X=\Phi
\end{array}
$$

- à la caractéristique de friction :

si l'on prend pour simplifier la tension $T=1$, la densité $\rho=1$ et la célérité $c=1$, on arrive aux deux équations :

$$
\begin{aligned}
\Psi & =\Phi-1 / 2 F(\Phi) \\
\text { et } \quad X & =1 / 2 F(\Phi)
\end{aligned}
$$

ou $F$ représente la caractéristique de friction (c'est donc la force appliquée par l'archet sur la corde). Si la pente maximum de $\mathrm{F}$ (qui est généralement la pente adhérence) est petite par rapport à l'impédance caractéristique de la corde, on obtient alors :

figure 3

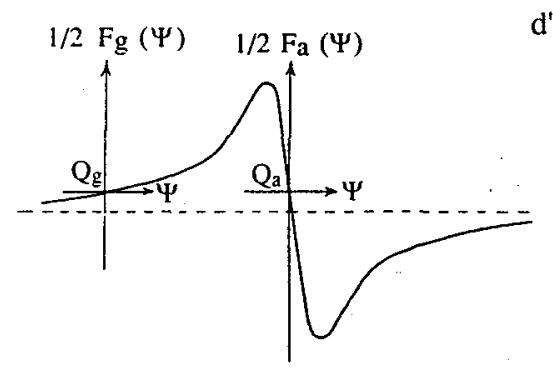

$$
1 / 2 \mathrm{~F}(\Phi) \approx 1 / 2 \mathrm{~F}(\Psi)
$$

$$
\text { d'où : } \Phi=\Psi+1 / 2 \mathrm{~F}(\Psi)
$$

Selon que $\Psi$, l'impulsion générée, et sa réflexion, passeront au niveau de l'archet lors d'une phase adhérence ou glissée, elles rencontreront des impédances différentes, comme cela est illustré sur la figure 3 où les deux points de fonctionnement pour le mouvement d' Helmholtz sont $\mathrm{Q}_{\mathrm{a}}$ (phase adhérence) et $\mathrm{Q}_{\mathrm{g}}$ (phase glissée). 
Ainsi, on peut distinguer deux cas :

a) l'impulsion passe deux fois lors de la phase adhérence de l'archet; l'équation s'écrit :

$$
\Delta \Psi=1 / 2 \mathrm{Fa}_{\mathrm{a}}(\Psi)-1 / 2 \mathrm{Fa}_{\mathrm{a}}(-\Psi)
$$

b) l'impulsion passe une fois en phase adhérence et sa réflexion lors de la phase glissée; l'équation est alors :

$$
\Delta \Psi=1 / 2 \mathrm{Fa}_{\mathrm{a}}(\Psi)-1 / 2 \mathrm{~F}_{\mathrm{g}}(-\Psi)
$$

La construction graphique (figure 4) permet de voir que, dans le premier cas, il ne peut y avoir aucune croissance ( $\Delta \Psi$ et $\Psi$ sont toujours de signes opposés) et qu'il n'existe pas de point stable pour l'impulsion générée alors que dans le deuxième cas il existe un point stable $Q_{s}$ (valeur de stabilisation de l'impulsion) et dans la zone hachurée la possibilité pour une perturbation faible de croître ( $\Delta \Psi$ et $\Psi$ de même signe) et de se stabiliser au point $Q_{S}$, ce qui est vérifié par la simulation et qui permet d'expliquer les observations expérimentales mentionnées au deuxième paragraphe.

\section{figure 4}
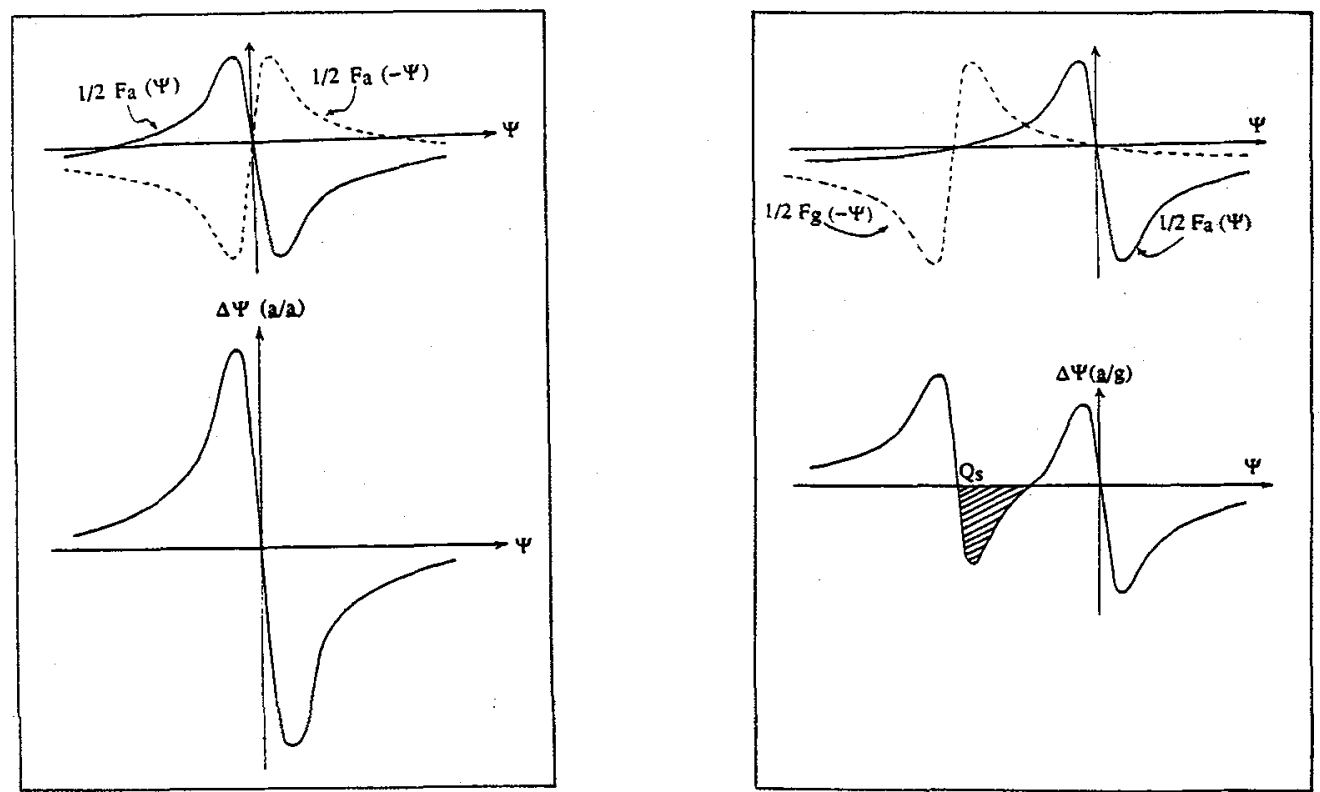

\section{REFERENCES}

[1] Weinreich, G. and Caussé, R., J. Acoust. Soc. Am. 89 (1991), 887-895.

[2] Raman, C.V., Indian Assoc. Cult. Sci. Bul. 15 (1918), 1-158.

[3] Krigar-Menzel, O. and Raps, A., Ann. Phys. Chem. 44 (1891), 613-629.

[4] McIntyre, M.E. and Woodhouse, J., Catgut Acoust. Soc. J. 42 (1984), 18-21.

[5] Caussé, R. and Weinreich, G., 13th International Congress on Acoustics, Belgrade Vol. 3 (1989), 83-86.

[6] Radulovic, S., Rapport 1053-S, Technische Universiteit Eindhoven (1990). 